\title{
Clinical value of ultrasonic elastography in diagnosing thyroid carcinoma.
}

\author{
Jie Lin* \\ Department of Ultrasound, Mianyang Central Hospital, Mianyang, PR China
}

\begin{abstract}
Objective: This study aims to explore the clinical value of ultrasonic elastography in diagnosing thyroid carcinoma.

Methods: Eighty thyroid carcinoma patients (100 nodules) admitted to a hospital were enrolled in this research. Routine ultrasonic testing and elastography were administered to all patients. The color and condition (benign or malignant) of the nodules were determined. The results were compared with pathogenic diagnosis of the operation. The specificity, sensitivity, and accuracy of the two test methods were computed and statistically compared.

Results: Pathological diagnosis of the operation suggested that among the 100 nodules, 70 were benign and 30 were malignant. The specificity, sensitivity, and accuracy of the routine ultrasonic check diagnosis were $\mathbf{5 0 . 0 0 \%}, \mathbf{5 3 . 3 3 \%}$, and $\mathbf{5 1 . 0 0 \%}$, respectively, whereas those of the ultrasonic elastography were $92.86 \%, 96.67 \%$ and $94.00 \%$, respectively. The former was significantly $(P<0.05)$ higher than the latter in all parameters.

Conclusion: Ultrasonic elastography showed a significantly higher critical value in diagnosing thyroid carcinoma and could accurately identify nodules as either benign or malignant. Hence, ultrasonic elastography is worth further critical promotion and application.
\end{abstract}

Keywords: Thyroid carcinoma, Ultrasonic elastography, Benign and malignant nodules, Clinical value. Accepted on October 24, 2017

\section{Introduction}

Ultrasonic elastography is a commonly-seen internal secretion malignant tumor [1]. In the same thyroid tissue, malignant nodules are much harder than benign tissues. Given its slow development and lack of clinical specificity, thyroid cancer can be hardly detected in the early period [2]. Currently, ultrasonic elastography has emerged as a new type of ultrasonoscopy technology with a wide array of applications [3]. In ultrasonic elastography, an external force is first exerted on tissues to cause distortion and deformation. Then, tissue elasticity is tested, and progression of the disease is judged based on the hardness of tissues and lesion [4]. In this study, 80 cases (100 tissues) admitted between March 2016 and August 2017 by the hospital the author works were studied and clinical value of ultrasonic elastography was examined.

\section{Materials and Methods}

\section{General materials}

Eighty thyroid carcinoma patients (100 nodules) admitted between March 2016 and August 2017 in a hospital were enrolled in this study. Among them, 50 were female patients (60 nodules), and 30 were male patients (40 nodules). The age range of the participants was 27-49 y, with an average of 47.79 $\pm 7.35 \mathrm{y}$. Their nodule diameters ranged from $5 \mathrm{~mm}$ to $30 \mathrm{~mm}$, with an average of $15.4 \pm 3.57 \mathrm{~mm}$. Tumors were significant in 51 cases and insignificant in 29 cases. All nodules were confirmed by operations to be accurately diagnosed. Some patients with diffuse lesion within nodes or with the crowd calcification diseases were excluded from this research. This study was approved by the hospital's ethnic committee. Patients were notified of the research purpose, and they provided informed consent.

\section{Methods}

The routine ultrasonic testing and ultrasonic elastography were administered to all patients. A Doppler color ultrasonic diagnostic apparatus was used to check patients. The linear array transducer frequency was between 4 and $13 \mathrm{MHz}$. The gray-scale ultrasound was used to test thyroid carcinoma. The test section was divided into cross and horizontal sections; the number, form, and size of nodules were observed and recorded in detail. Ultrasonic elastography was then performed among patients. The sampling frame should be larger than the range of the focus. The hand-held probe underwent microvibration in the position of the focus. The voltage index on the instrument screen was maintained between 3 and 4 . The gray-scale and elastic images were observed. The hardness of the focus and the surrounding tissues was compared. Green denotes the average hardness of tissues within the sampling frame; blue means the hardness of tissues higher than the average hardness; 
and red signifies the hardness of tissues smaller the average hardness. Two experienced physicians carried out the joint survey to guarantee accuracy and effectiveness of the test.

\section{Observational indexes}

The color of the focus was observed to determine whether a nodule was benign or malignant. The observation results were compared with the pathological diagnosis to calculate the specificity, sensitivity, and accuracy of the two test methods. The test results were then statistically compared.

A grade system was devised in identifying the focus and tissue color in the ultrasonic elastography: grade 0 , blue, red, green, or blue and red; grade I, green; grade II, blue; grade III, blue and green; and grade IV, blue. The elasticity of benign nodules ranged from grade 0 to grade $\mathrm{II}$, whereas that of malignant nodules ranged from grade III to grade IV.

\section{Statistical processing}

Parameters (specificity, sensitivity, and accuracy) of the methods were analysed using SPSS 22.0 and were expressed in percentages. Pearson's chi-square test ( $\chi^{2}$ test) was performed, and statistically significant difference was considered at $\mathrm{P}<0.05$.

\section{Results}

\section{Pathogenic diagnosis results}

Pathological diagnosis suggested that, among 100 nodules, 70 (70\%) were benign and $30(30 \%)$ were malignant.

\section{Specificity, sensitivity, and accuracy of the ultrasonic elastography}

As shown in Table 1, the routine ultrasonic testing achieved a specificity of $50.00 \%(35 / 70)$, a sensitivity of $53.33 \%(16 / 30)$, and an accuracy of $51.00 \%(51 / 100)$.

Table 1. Specificity, sensitivity, and accuracy of the routine ultrasonic testing.

\begin{tabular}{llll}
\hline Pathological diagnosis & \multicolumn{2}{l}{ Routine ultrasonic testing } & Total \\
\cline { 2 - 3 } & Benign & Malignant & \\
\cline { 2 - 3 } Benign & 35 & 35 & 30 \\
\hline Malignant & 14 & 16 & 30 \\
\hline Total & 49 & 51 & 100 \\
\hline
\end{tabular}

\section{Ultrasonic elastography diagnosis results}

In ultrasonic elastography (Table 2), 66 out of $100(66 \%)$ nodules were benign, and $34(34 \%)$ were malignant nodules.

Table 2. Ultrasonic elastography results of 100 nodules.

\begin{tabular}{lll}
\hline Elasticity & Number of cases $(\mathrm{n})$ & Percentage (\%) \\
\hline
\end{tabular}

\begin{tabular}{lcc}
\hline Grade 0 & 30 & 30.00 \\
\hline Grade I & 16 & 16.00 \\
\hline Grade II & 20 & 20.00 \\
\hline Grade III & 14 & 14.00 \\
\hline Grade IV & 20 & 20.00 \\
\hline
\end{tabular}

\section{Specificity, sensitivity, and accuracy of ultrasonic elastography}

Ultrasonic elastography (Table 3) obtained a specificity of $92.86 \%(65 / 70)$, a sensitivity of $96.67 \%$ (29/30), and an accuracy of $94.00 \%(94 / 100)$.

Table 3. Specificity, sensitivity, and accuracy of ultrasonic elastography diagnosis results.

\begin{tabular}{llll}
\hline Pathogenic diagnosis & \multicolumn{2}{l}{ Routine ultrasonic testing } & Total \\
\cline { 2 - 3 } & Benign & Malignant & \\
\hline Benign & 65 & 5 & 70 \\
\hline Malignant & 1 & 29 & 30 \\
\hline Total & 66 & 34 & 100 \\
\hline
\end{tabular}

\section{Comparison of routine ultrasonic and ultrasonic elastography testing results}

As shown in Table 4, the specificity, sensitivity, and accuracy of ultrasonic elastography diagnosis results were significantly higher than those of the routine ultrasonic testing $(\mathrm{P}<0.05)$.

Table 4. Comparison of the routine ultrasonic and ultrasonic elastography testing results.

\begin{tabular}{llll}
\hline Test method & Specificity & Sensitivity & Accuracy \\
\hline $\begin{array}{l}\text { Routine ultrasonic } \\
\text { testing }\end{array}$ & $50.00 \%(35 / 70)$ & $53.33 \%(16 / 30)$ & $51.00(51 / 100)$ \\
\hline $\begin{array}{l}\text { Ultrasonic } \\
\text { elastography }\end{array}$ & $92.86 \%(65 / 70)$ & $96.67 \%(29 / 30)$ & $94.00(94 / 100)$ \\
\hline$X^{2}$ value & 31.5000 & 15.0222 & 46.3699 \\
\hline$P$ value & 0.0000 & 0.0001 & 0.0000 \\
\hline
\end{tabular}

\section{Discussion}

Ultrasonic elastography is a type of ultrasonoscopy reflecting lesion elastic information before and after imposition of an external force. After an external force is exerted on the tissues, the normal soft tissues undergo a higher degree of deformation as compared with the hard tissues [5]. Lesion changes before and after an external force is exerted, which shows the degree of lesion hardness. Lesion hardness is regarded as one of major parameters to evaluate the nature of lesion. For example, lowecho lesion diagnosis is a difficult but high-elasticity procedure; this method can be used in the diagnosis of adipose tissues [6]. Conversely, ultrasonic elastography provides elasticity information of biological tissues for accurate 
evaluation of diseases. Different tissues have varying elasticity coefficients. When an external force is imposed or an alternating vibration occurs, tissues are changed morphologically. During testing, signals of the tested body in different periods of time are collected and analysed using autocorrelation [7]. Gray-scale imaging or color-coding imaging is then carried out. If the imposed external force is the same as before, then the higher the elasticity coefficient is, the smaller the morphological change will be. On the contrary, the smaller the elasticity coefficient is, the larger the morphological change will be. This generalization suggests that deformation of normal soft tissues is much more significant than that of hard tumor tissues [8]. Ultrasonic elastography aims to generate different morphological changes based on differences between the tumor or lesion area and the surrounding normal tissues in terms of the elasticity coefficient. Meanwhile, color coding is adopted to show such morphological changes, thereby enabling judgment of elasticity of lesion tissues and prediction of potential lesions.

Thyroid cancer is a clinically common disease whose morbidity has increased in recent years [9]. Pathogenesis of thyroid cancer is often unobvious and can be divided into benign and malignant lesions. Immediate and early discovery, diagnosis, and identification of thyroid cancer can provide reliable references for clinical treatment and rehabilitation of patients with this disease. The routine ultrasonic testing is the top choice for thyroid cancer, where in the lesion undergoes $2 \mathrm{D}$ gray-scale ultrasonic testing and Doppler color ultrasonic diagnosis. Despite its low specificity, sensitivity, and accuracy, $2 \mathrm{D}$ gray-scale ultrasonic testing remains an effective evaluation tool in determining benign and malignant thyroid lesions [10]. However, ultrasonic elastography has emerged as a new diagnosis approach. This method's judgment of the hardness of relevant tissues, including thyroid glands, can help diagnose the condition (benign or malignant) of tissues. Furthermore, the diagnostic specificity, sensitivity, and accuracy of this procedure are high, which can fully compensate the shortage of the routine ultrasonic testing of commonly-seen diseases.

Results suggested that the specificity, sensitivity, and accuracy of the routine ultrasonic testing were $50.00 \%, 55.56 \%$, and $51.43 \%$, respectively, whereas those of ultrasonic elastography were $92.86 \%, 96.67 \%$, and $94.00 \%$, respectively. The latter was significantly higher $(\mathrm{P}<0.05)$ than the former in all parameters. These findings highly coincide with the research of Gao, who reported the same percentages in all categories for routine ultrasonic testing. However, these percentages are significantly lower than those of ultrasonic elastography, with $92.31 \%, 100.00 \%$, and $94.28 \%$, respectively. Similarly, the latter was significantly higher $(\mathrm{P}<0.05)$ than the former. This comparative analysis indicates that ultrasonic elastography could better diagnose thyroid cancer, with a higher specificity, sensitivity, and accuracy, as compared with the routine ultrasonic testing. Overall, ultrasonic elastography could accurately identify and diagnose whether thyroid nodules are benign or malignant and could provide reliable references for clinical diagnosis and treatment.

\section{Conclusion}

Ultrasonic elastography has a higher clinical value in diagnosing thyroid cancer and can accurately identify benign and malignant nodules. Thus, ultrasonic elastography is worth further promotion and application.

\section{References}

1. Al-Sharaky DR, Younes SF. Sensitivity and specificity of galectin-3 and glypican-3 in follicular-patterned and other thyroid neoplasms. J Clin Diagn Res 2016; 10: 6-10.

2. Lu HB, Wang XY, Zhang WB, Liu YY. Two novel pyranketone derivatives: synthesis, crystal structures and inhibiting thyroid adenoma cell activity in vitro. Lat Am J Pharm 2017; 36: 1289-1293.

3. Zhu F, Shen YB, Li FQ, Fang Y, Hu L, Wu YJ. The effects of hashimoto thyroiditis on lymph node metastases in unifocal and multifocal papillary thyroid carcinoma. Medicine (Baltimore) 2016; 95: 2674.

4. Han C, Wei X. Novel biomarkers to determine neonates with necrotizing enterocolitis. Biomed Res India 2017; 28: 6002-6006.

5. Barbesino G, Goldfarb M, Parangi S, Yang J, Ross DS, Daniels GH. Thyroid lobe ablation with radioactive iodine as an alternative to completion thyroidectomy after hemithyroidectomy in patients with follicular thyroid carcinoma: long-term follow-up. Thyroid 2012; 22: 369-376.

6. Ruan FC, Dai J, Yan QH, Lu Y. Altered metabolic behavior of derivatives of herbal compounds used for treatment of carcinoma of mouth. Lat Am J Pharm 2017; 36: 1730-1735.

7. Kawada N, Tanaka S. Elastography for the pancreas: Current status and future perspective. World J Gastroenterol 2016; 22: 3712-3724.

8. Sigrist RMS, Liau J, Kaffas AE, Chammas MC, Willmann JK. Ultrasound elastography: review of techniques and clinical applications. Theranostics 2017; 7: 1303-1329.

9. Li LJ, Zeng H, Ou B, Luo BM, Xiao XY, Zhong WJ, Zhao XB, Zhao ZZ, Yang HY, Zhi H. Ultrasonic elastography features of phyllodes tumors of the breast: a clinical research. PLoS One 2014; 9: 85257.

10. Tavakoli GA, Movahedi MM, Reza M, Hassani K. A study on performance and safety test of infusion pump devices. Biomed Res India 2017; 28: 5179-5181.

\section{*Correspondence to}

Jie Lin

Department of Ultrasound

Mianyang Central Hospital

PR China 